\title{
Hot surface ignition of stoichiometric hydrogen-air mixtures
}

\author{
J. Melguizo-Gavilanes*, L.R. Boeck, R. Mével, J.E. Shepherd \\ Graduate Aerospace Laboratories, California Institute of Technology (GALCIT), Pasadena, CA, \\ USA
}

\begin{abstract}
Hot surface ignition is relevant in the context of industrial safety. In the present work, two-dimensional simulations with detailed chemistry, and study of the reaction pathways of the buoyancy-driven flow and ignition of a stoichiometric hydrogen-air mixture by a rapidly heated surface (glowplug) are reported. Experimentally, ignition is observed to occur regularly at the top of the glowplug; numerical results for hydrogen-air reproduce this trend, and shed light on this behavior. The simulations show the importance of flow separation in creating zones where convective losses are minimized and heat diffusion is maximized, resulting in the critical conditions for ignition to take place.
\end{abstract}

Key words: hot surface ignition, industrial safety, interferometry, numerical simulation, hydrogen

${ }^{*}$ Corresponding author: josuemg@caltech.edu 


\section{Introduction}

Ignition of combustible atmospheres by hot surfaces is a common issue in industrial safety. Determining critical conditions for ignition in terms of surface size and temperature are essential in order to evaluate the potential of an ignition hazard.

Classical experimental work on hot surface ignition includes that of Coward and Guest [1], and Kutcha [2]. The former investigated the effect of material (e.g catalytic and non-catalytic surfaces) on ignition thresholds, whereas the latter extended this work to study the effect of variations in size and geometry. The impact of their results was limited by their inability to measure flow velocity and composition during the ignition event. An extensive review of more recent studies is given by Brabauskas [3]. Experimental work done by Boettcher [4] using a glow plug found the ignition temperature for n-Hexane to be essentially insensitive to composition away from flammability limits. Analytical studies have been performed by Gray [5] who investigated the effect of surface to volume ratio, and more recently Laurendeau [6] in which a simple model is proposed to estimate the minimum ignition temperature.

Some numerical efforts in this area are due to Kumar [7] who developed a onedimensional model to study hydrogen ignition, and the two-dimensional steady simulations of Adler [8] in which the problem of a circular hot spot in contact with reactive mixture was analyzed. Boettcher [4] carried out simulations to examine predicting lower flammability limits with tabulated chemistry as well as studying the effect of hot surface area on ignition temperature using one-step and detailed chemical reactive models [9].

None of the previous work has been concerned with analyzing in detail the flow field in the vicinity of the hot surface. For an accurate numerical prediction of this flow, it is necessary to solve the conservation equations together with transport of chemical species on a mesh small enough to capture the thermal and hydrodynamic boundary layer surrounding the hot surface. The wide range of temporal and spatial scales involved, as well as the size of detailed chemical kinetic mechanisms pose 
significant computational challenges. Hydrogen is one of the fuels for which the chemistry is reliably known -all reaction rates have been experimentally measured-, and the detailed kinetic mechanism is of a reasonable size to simulate realistic geometries. A two-dimensional numerical simulation of the transient viscous flow and reaction of combustible atmospheres using a detailed hydrogen oxidation mechanism is presented. Special attention is given to the near-wall induced buoyancy flow, and flow separation to gain insight on the dynamics, time and location of the ignition event.

\section{Physical model and computational methodology}

\subsection{Governing equations}

The motion, transport and chemical reaction in the gas surrounding the glowplug are modeled using the variable-density reactive Navier-Stokes equations with temperature-dependent transport properties.

$$
\begin{gathered}
\partial_{t}(\rho)+\nabla \cdot(\rho \mathbf{u})=0 \\
\partial_{t}(\rho \mathbf{u})+\nabla \cdot(\rho \mathbf{u u})=-\nabla p+\nabla \cdot \tau+\rho \mathbf{g} \\
\partial_{t}\left(\rho Y_{i}\right)+\nabla \cdot\left(\rho \mathbf{u} Y_{i}\right)=-\nabla \cdot \mathbf{j}_{\mathbf{i}}+\dot{\omega}_{i} \\
\partial_{t}\left(\rho h_{s}\right)+\nabla \cdot\left(\rho \mathbf{u} h_{s}\right)=-\nabla \cdot \mathbf{j}_{\mathbf{q}}+\dot{q}_{\mathrm{chem}} \\
p=\rho \bar{R} T, \quad \tau=\mu\left[\nabla \mathbf{u}+(\nabla \mathbf{u})^{T}\right]-\frac{2}{3} \mu(\nabla \cdot \mathbf{u}) \mathbf{I}
\end{gathered}
$$

In Eqs. (1)-(5), $\rho, p$ and $T$ are the gas density, pressure and temperature, $\mathbf{u}$ is the velocity vector, $h_{s}$ is the mixture sensible enthalpy, $\mathbf{g}$ is the gravitational acceleration, $Y_{i}$ is the mass fraction of species, $\mathbf{j}_{\mathbf{i}}$ is the species diffusion flux, $\dot{\omega}_{i}$ represents the rate of production/consumption of species, $\mathbf{j}_{\mathbf{q}}$ is the heat flux, $\dot{q}_{\mathrm{chem}}=-\sum_{i=1}^{N} \Delta h_{f, i}^{o} \dot{\omega}_{i}$ is the rate of conversion of chemical into thermal energy, $\Delta h_{f, i}^{o}$ is the enthalpy of formation of species, $\bar{R}$ is the specific gas constant, $\mu$ is the mixture viscosity, and $\mathbf{I}$ is the identity matrix. Radiation is neglected in the current numerical model. 
The species diffusion term, $\mathbf{j}_{\mathbf{i}}$, uses Fick's law for binary mixtures. For multicomponent mixtures where one component is present in large amounts (i.e. $\mathrm{N}_{2}$ for combustion in air) all other species may be treated as trace species. Writing the binary diffusion coefficient with respect to $\mathrm{N}_{2}$ yields:

$$
\mathbf{j}_{\mathbf{i}}=-\rho D_{i} \nabla Y_{i}, \text { with } D_{i}=D_{j, N_{2}}
$$

where $D_{i}$ is the effective diffusion coefficient. In Eq. 6, thermodiffusion or Soret effect has been neglected.

We solve the mass conservation equation, Eq. 1, and only for $N-1$ species equations, Eq. 3. The last species mass fraction, $\mathrm{N}_{2}$, is obtained by writing $Y_{N_{2}}=$ $1-\sum_{i=1}^{N-1} Y_{i}$ and absorbs all inconsistencies introduced by Fick's law. This error is negligible when the last species, $Y_{N_{2}}$, is in a high concentration as is the case for combustion in air [10].

The heat flux $\mathbf{j}_{\mathbf{q}}$ includes the effect of sensible enthalpy transport by diffusion

$$
\mathbf{j}_{\mathbf{q}}=-\frac{\kappa}{c_{p}} \nabla h_{s}+\sum_{i=1}^{N-1} h_{s, i}\left(\mathbf{j}_{\mathbf{i}}+\frac{\kappa}{c_{p}} \nabla Y_{i}\right)
$$

where $\kappa$ and $c_{p}$ are the mixture averaged thermal conductivity and specific heat, respectively. In Eq. 7, the Dufour effect (i.e. energy flux due to a concentration gradient) has not been taken into account [11]. Substituting Eq. 6 into 7 yields:

$$
\mathbf{j}_{\mathbf{q}}=-\frac{\kappa}{c_{p}} \nabla h_{s}+\sum_{i=1}^{N-1} h_{s, i}\left(1-\frac{1}{\mathrm{Le}_{i}}\right) \frac{\kappa}{c_{p}} \nabla Y_{i}
$$

where $\mathrm{Le}_{i}=\kappa /\left(c_{p} \rho D_{i}\right)$ is the Lewis number of species $i$. The second term on the right hand side of Eq. 8 vanishes if the Lewis numbers of all species are assumed to be unity. This approximation is common in combustion codes but is not justified in the present case [10].

The equations above are integrated in two dimensions using the Open source Field Operation And Manipulation (OpenFOAM) toolbox [12]. The spatial discretization of the solution domain is performed using finite volumes. Specifically, the convective terms were discretized using a second order, bounded TVD scheme; the mass fractions were discretized using a linear centered scheme for scalars bounded 
between zero and one. The diffusion terms were discretized using the linear centered scheme together with a second order, conservative scheme for the evaluation of the surface normal gradients. The linear systems that result from the discretization of the governing equations are solved through iterative techniques [13]. The PBiCG (Preconditioned Biconjugate Gradient) method is used for all linear systems including the chemical source terms preconditioned through the DILU (Diagonal Incomplete-LU) technique, whereas the Poisson equation for pressure is solved using the PCG (Preconditioned Conjugate Gradient) preconditioned by the DIC (Diagonal Incomplete Cholesky). The pressure-velocity coupling is achieved using the PIMPLE (PISO+SIMPLE) algorithm [14]. Finally, the time-step is dynamically adapted during the course of the computation based on a specified Courant number to ensure stability of the numerical scheme [15]. In the current study the Courant number used is 0.2 .

\subsection{Chemical and transport models}

The chemistry is modeled using Mével's detailed mechanism for hydrogen oxidation which includes 9 species and 21 reactions. This mechanism has been extensively validated, and reproduces flame speeds and ignition delay times to a reasonable degree of accuracy over a wide range of concentrations $[16,17]$. Among the available alternatives, Mével's model provides a sound middle ground. It is not the fastest nor the slowest regarding ignition delay time predictions, which is the quantity of interest in the current study. The Sutherland Law, modified Eucken relation and JANAF polynomials are used to account for the functional temperature dependence of mixture viscosity $(\mu)$, thermal conductivity $(\kappa)$ and specific heat $\left(c_{p}\right)$, respectively. Species diffusivities are computed using Cantera [18]; a constant non-unity Lewis number, $\mathrm{Le}_{i}$, is specified for each species. This is a convenient approximation since $\mathrm{Le}_{i}$ values are essentially constant and vary in small amounts across flame fronts $[10]$.

\subsection{Domain, initial and boundary conditions}

The geometry simulated corresponds a combustion vessel of $11.43 \mathrm{~cm} \mathrm{x} 16.51 \mathrm{~cm}$ with a glowplug of height $9.3 \mathrm{~mm}$, and diameter $5.1 \mathrm{~mm}$ located in the center of the 
bottom plane. There are approximately 200,000 cells in the 2D-axisymmetric computational domain. Cells are compressed near the wall of the glow plug, with a minimum cell size of $80 \mu \mathrm{m}$ to properly resolve the thermal and hydrodynamic boundary layers. The initial conditions are $p_{o}=101 \mathrm{kPa}, T_{o}=300 \mathrm{~K}, \mathbf{u}_{\mathbf{o}}=(0,0,0) \mathrm{m} / \mathrm{s}$, and mass fractions corresponding to a stoichiometric mixture $\left(Y_{\mathrm{H}_{2}}=0.0283, Y_{\mathrm{O}_{2}}\right.$ $\left.=0.2264, Y_{\mathrm{N}_{2}}=0.7453\right)$. No-slip boundary condition and constant temperature $T_{\text {wall }}=T_{o}$ is imposed on the vessel walls, and on the glowplug surface, a prescribed temperature ramp given by $T(t)=T_{o}+r t$ with $r=220 \mathrm{~K} / \mathrm{s}$.

\section{Validation and comparison with experiments during heating}

To test the heat transfer and fluid mechanics in the numerical model, an experimental temperature field (obtained via interferometry) of the heating of a stoichiometric $\mathrm{H}_{2}$-air mixture was compared with a simulated field. Details on the experimental setup, and diagnostics used can be found in Melguizo-Gavilanes et al. [19]. Experimentally, two heating rates were used $18 \mathrm{~K} / \mathrm{s}$ and $190 \mathrm{~K} / \mathrm{s}$, as it was observed that imposing a slow heating rate resulted in more uniform heating of the glowplug. Numerically, as explained in the previous section, a uniform heating ramp of $220 \mathrm{~K} / \mathrm{s}$ is used. Imposing a fast heating ramp results in a less expensive computation as it takes less time to reach the critical temperature for ignition to occur. In principle, the rate at which the gas is heated may affect the flow field, as well as the time at which the ignition event takes place. The effect of using slow and fast heating ramps experimentally, and their influence in the resulting flow field is quantified in this section. The instantaneous experimental fields were taken $\sim$ $32 \mathrm{~s}$ and $3 \mathrm{~s}$ after heating corresponding to a glowplug surface temperature of 876 $\mathrm{K} \pm 42 \mathrm{~K}$ and $870 \mathrm{~K} \pm 42 \mathrm{~K}$, for $18 \mathrm{~K} / \mathrm{s}$ and $190 \mathrm{~K} / \mathrm{s}$, respectively. The surface temperature was determined experimentally using pyrometry. The numerical fields were taken after $2.6 \mathrm{~s}$ of heating, or $\mathrm{T}_{\text {surf }}=872 \mathrm{~K}$, in order to match as closely as possible the experimental values for both heating ramps.

Figure 1 shows the experimental, simulated and a side-to-side comparison of the temperature fields, together with temperature samples at different heights in 

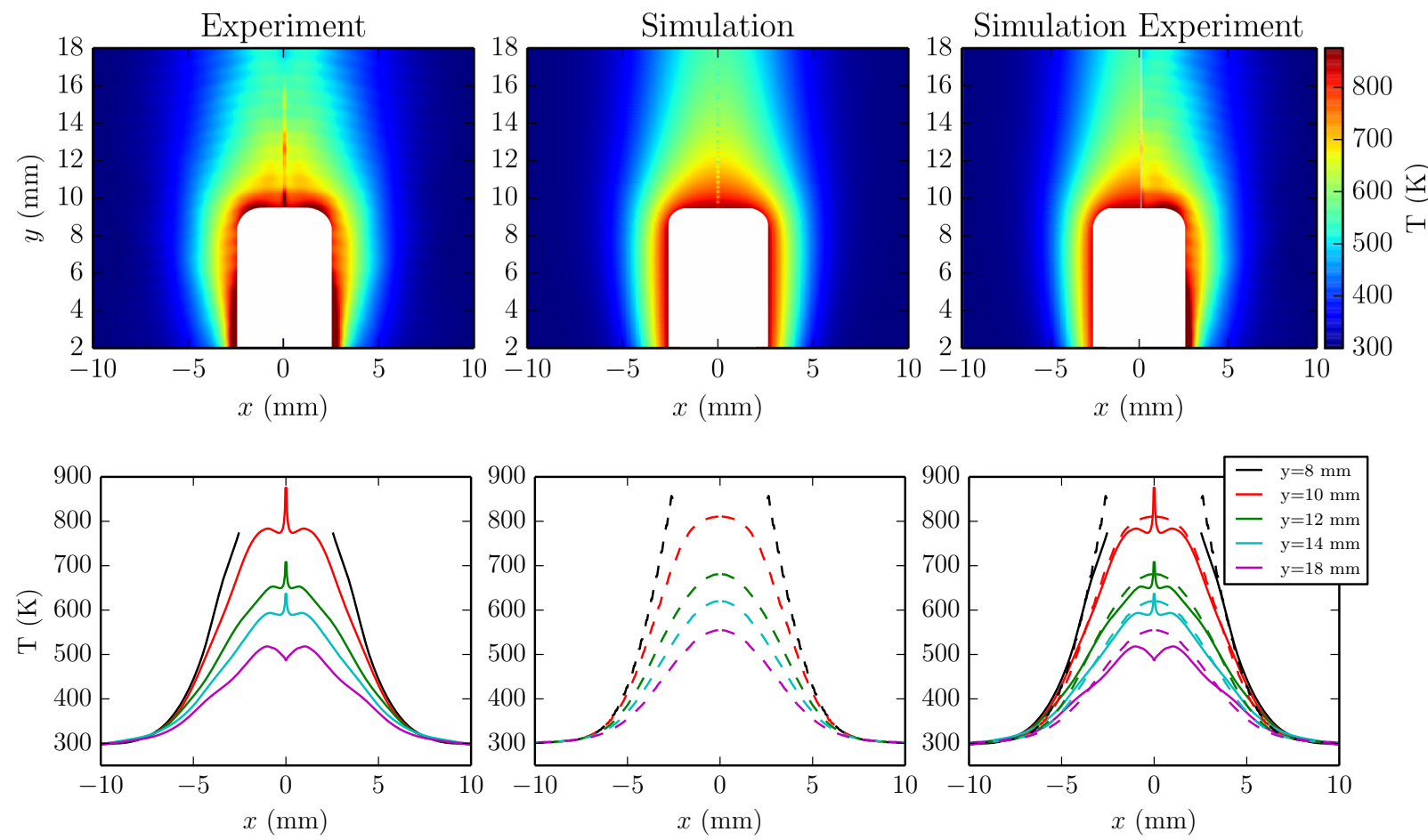

Figure 1: Fast heating - $190 \mathrm{~K} / \mathrm{s}$. Top: comparison of experimental and numerical temperature fields during heating - glowplug surface temperature $870 \mathrm{~K}$. Bottom: comparison of experimental (solid lines) and numerical (dashed lines) temperature profiles at different heights in the flow field: $\mathrm{y}=8,10,12,14,18 \mathrm{~mm}$.

the flow field for a heating rate of $190 \mathrm{~K} / \mathrm{s}$. Good agreement is observed in the thickness of the thermal plume and the temperature samples at all heights examined, except for an artifact present in the experimental traces at the centerline, and the underprediction of the wall temperature in the experimental trace at $y=8 \mathrm{~mm}$. These discrepancies could be attributed to an incipient asymmetry in the thermal plume caused by non-uniformities in the heating of the glowplug, and the known limitations of the postprocessing of the experimental optical phase difference fields obtained using interferometry. Errors are accumulated from the outer edges of the image towards the axis of symmetry, and near solid boundaries while performing the inversion of the Abel transform [20]; a crucial and necessary step to obtain the experimental temperature field.

Figure 2 shows the same fields and traces described in Fig. 1 but for a heating rate of $18 \mathrm{~K} / \mathrm{s}$. The artifact at the centerline is not as pronounced in the temperature 

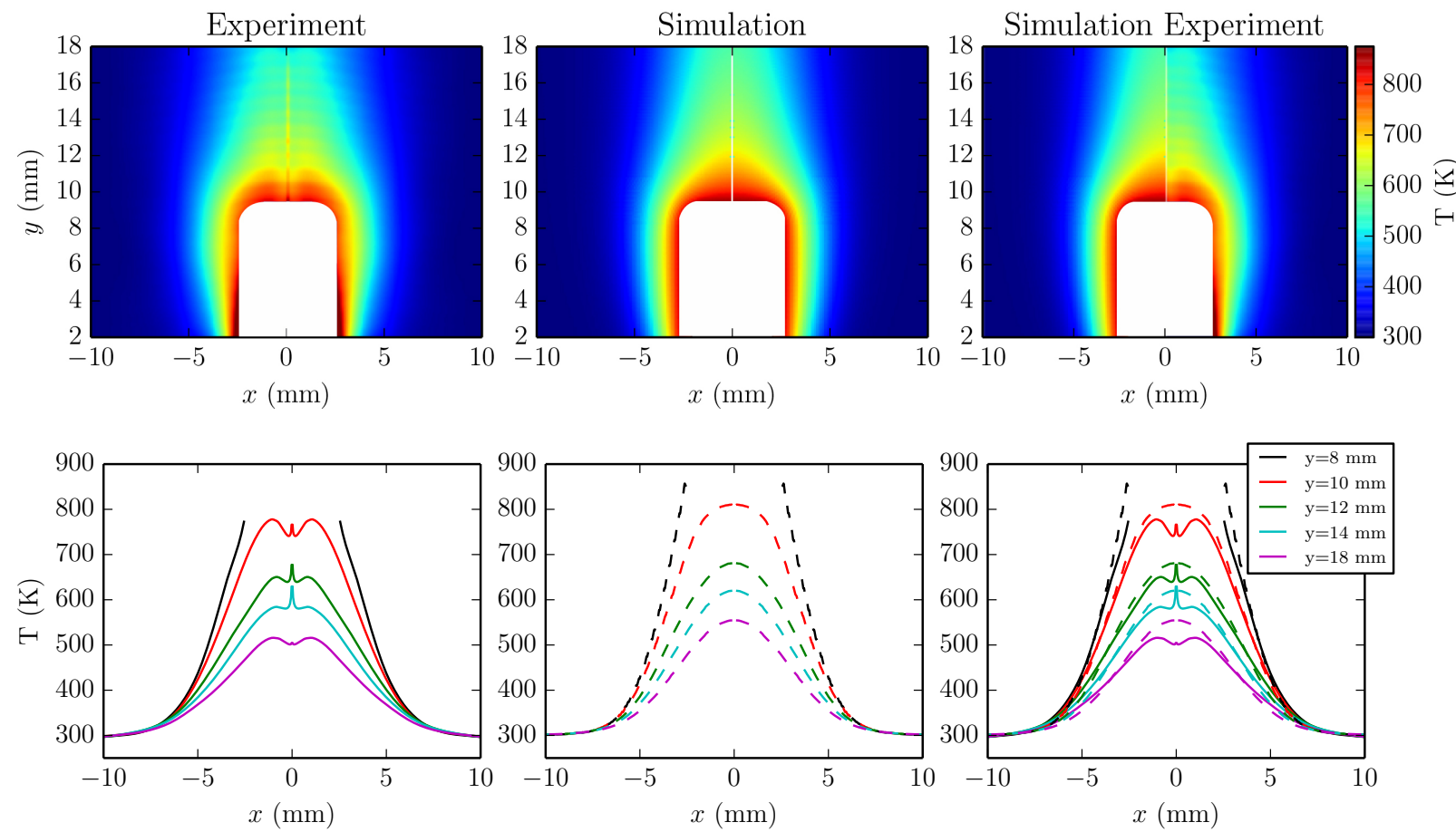

Figure 2: Slow heating - $18 \mathrm{~K} / \mathrm{s}$. Top: comparison of experimental and numerical temperature fields during heating - glowplug surface temperature $870 \mathrm{~K}$. Bottom: comparison of experimental (solid lines) and numerical (dashed lines) temperature profiles at different heights in the flow field: $\mathrm{y}=8,10,12,14,18 \mathrm{~mm}$.

traces and the temperature fields seem to be smoother, however the wall temperature continues to be underpredicted. Once again, very good agreement is obtained when comparing both temperature fields, and the samples taken at different heights.
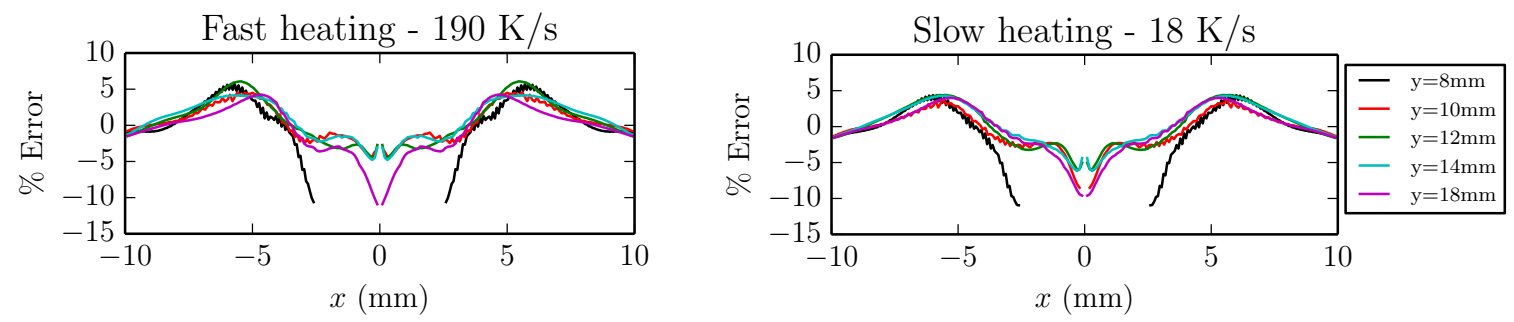

Figure 3: Error between experimental and predicted fields at different heights in the flow field: y $=8,10,12,14,18 \mathrm{~mm}$, for fast and slow heating.

To quantify the discrepancy between the simulated and the experimental fields, the percentage of error was computed taking the simulated fields as a reference (see Fig. 3). Negative values of error correspond to higher predicted temperatures, 
whereas positive values correspond to lower temperature predictions. Errors of less than $5 \%$ were obtained across the viewing window/numerical domain for both heating rates. Notably, even in the regions where the experimental postprocessing techniques are known to perform worst, i.e. centerline and near walls, the maximum error is of only $10 \%$. These results validate the numerical methodology used, and provide evidence of the minor role played by the heating rate in the evolution of the thermal plume and flow field.

\section{Ignition Results}

\subsection{Experimental Results}

A total of six experiments were performed at stoichiometric conditions, with three repetitions for each heating rate. The results are summarized in Fig. 4. An ignition threshold of $1052 \mathrm{~K} \pm 52 \mathrm{~K}$, and $1028 \mathrm{~K} \pm 53 \mathrm{~K}$ was obtained for $18 \mathrm{~K} / \mathrm{s}$ and $190 \mathrm{~K} / \mathrm{s}$ respectively. Sources of uncertainty in the ignition surface temperature reported in the experiments include wavelength dependence on emmisivity, noise and calibration errors, and glowplug surface temperature non-uniformities. The observed shift in ignition threshold between the fast and slow heating rate cases is likely due to pyrometer field of view. There are regions on the surface of the heated element where the temperature is higher (this is more pronounced when the fast heating is used) than in the location recorded by the pyrometer. One could also argue that the $24 \mathrm{~K}$ difference in ignition thresholds between heating rates simply means that for stoichiometric hydrogen-air the heating rate plays a minor role in determining the ignition threshold of the mixture. This is in contrast with hydrocarbons ( $n$-hexane) where the rate at which the mixture is heated can influence the ignition threshold due to low temperature pathways as shown by Menon et al. [21]. Such effects may be important for very lean hydrogen-air mixtures at low heating rates, however we did not examine these situations in the present study.

Figure 5 shows a time sequence of a typical ignition event. The time interval between frames is $100 \mu \mathrm{s}$. The formation of an ignition kernel at the top of the glowplug is clearly visualized, which subsequently turns into a flame that propagates radially outwards preferentially along the hotter thermal plume. Note that some 


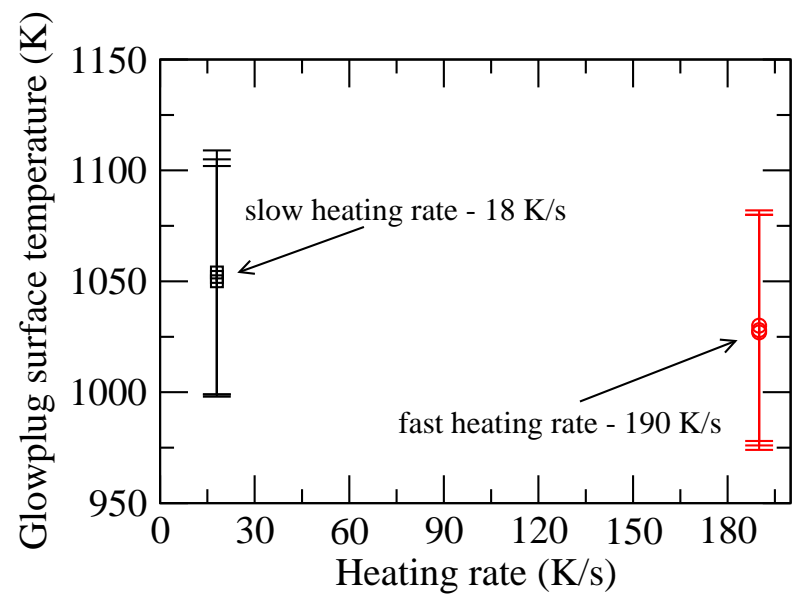

Figure 4: Experimental ignition threshold obtained for stoichiometric hydrogen-air mixture at $p_{o}$ $=101 \mathrm{kPa}$ and $T_{o}=300 \mathrm{~K}$ using slow and fast heating rates.
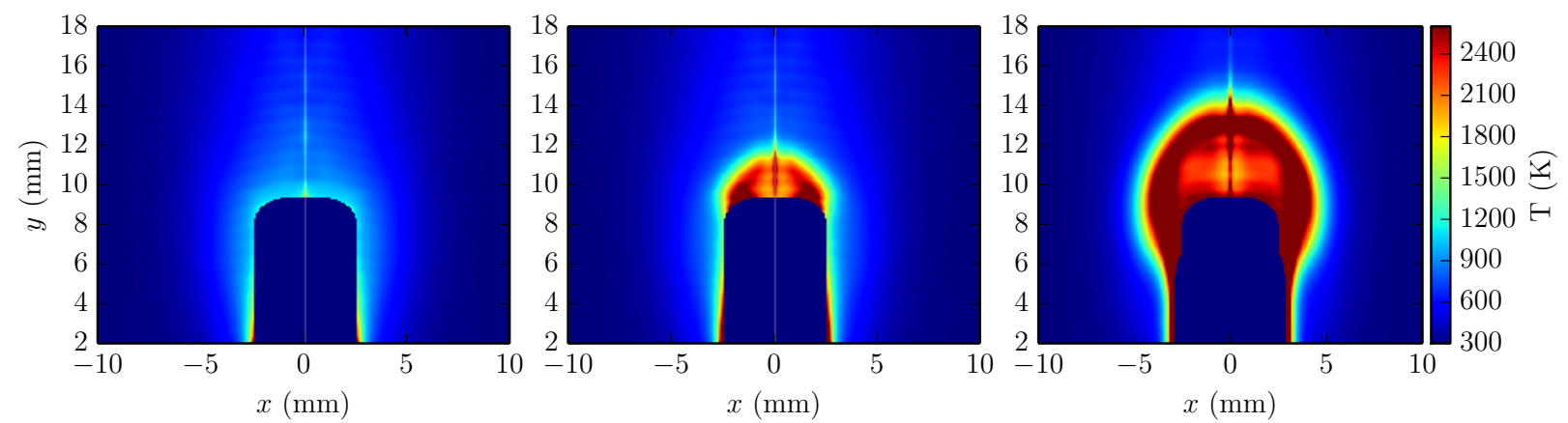

Figure 5: Experimental temperature fields for ignition experiments at $p_{o}=101 \mathrm{kPa}, T_{o}=300$ $\mathrm{K}$ and stoichiometric hydrogen-air mixture using a slow heating rate. The time interval between frames is $100 \mu \mathrm{s}$.

of the technical difficulties discussed in section 3 become more apparent with the presence of a flame, namely, increased noise at the axis of symmetry, near sharp gradients and solid boundaries. Additionally, at this point, our postprocessing is further affected by the abrupt expansion and high flame speeds characteristic of stoichiometric hydrogen-air mixtures. Pressure waves emanating from the initial ignition/flame kernel, affect the thermodynamic state of fresh unburnt reactants present in the vessel. The end result is the presence of substantial uncertainty in the computed temperature field as the postprocessing relies on a fixed initial pressure 
and the use of the ideal gas law to compute the temperature. We are therefore only using this visualization qualitatively to show the ignition event, flame kernel formation and early stages of flame propagation. In the next subsection, we examine ignition in detail by means of numerical simulations.

\subsection{Numerical Results}

\subsubsection{Flow structure}

A detailed analysis of the flow field during the ignition event has been performed to identify important features in the flow such as thermal and hydrodynamic boundary layers, flow separation, thermal plume temperature, velocity distributions and chemical activity.

Figure 6 (Top) shows the temperature $(\mathrm{T})$ and velocity magnitude $\left(u_{\text {mag }}\right)$ fields obtained $83 \mu$ s before ignition, or alternatively, $2.899575 \mathrm{~s}$ after the start of heating. Temperature filled contours taken every $30 \mathrm{~K}$ from $400 \mathrm{~K} \leq T \leq 938 \mathrm{~K}$, and velocity vectors showing clearly the buoyancy driven flow induced by the glowplug. Figure 6 (Bottom) shows plots of the spatial distribution of velocity $\left(u_{x}, u_{y}\right.$, and $\left.u_{m a g}\right)$, and temperature at two locations from the surface of the glowplug. The left plot displays the vertical spatial distribution starting at $(x=0 \mathrm{~mm}, y=9.3 \mathrm{~mm})$-immediately above the hot surface, whereas the right plot displays the horizontal spatial distribution starting at $(x=2.55 \mathrm{~mm}, y=5 \mathrm{~mm})$-on the side of the glowplug. The origin of the coordinate system $(x=0 \mathrm{~mm}, y=0 \mathrm{~mm})$ is located at the bottom on the vertical centerline of the glowplug. In the vicinity of the hot surface a thermal boundary layer can be observed, the buoyancy of the heated gas induces a flow in the gas surrounding the glowplug, and a thermal plume is created above the glowplug. Note that in the separated region (top of the glowplug) there is a thicker thermal layer (shown in more detail in section 3). The thermal plume is outlined by the outermost temperature contour at $T=400 \mathrm{~K}$. The velocity magnitude field and velocity vectors show the flow structure near the glowplug. Parcels of fresh cold gas enter the thermal boundary layer from below and heat up slowly as they travel upward in close proximity to the wall. Once the parcels of gas reach the upper right/left corner of the glowplug, the flow separates, creating a region at the top of 


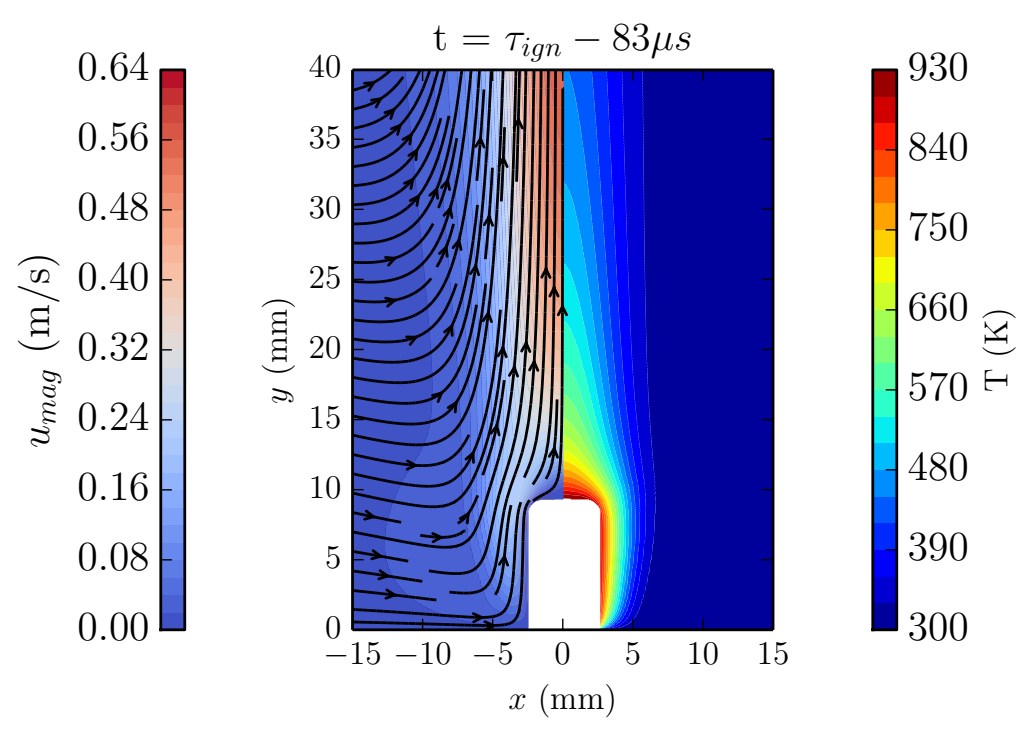

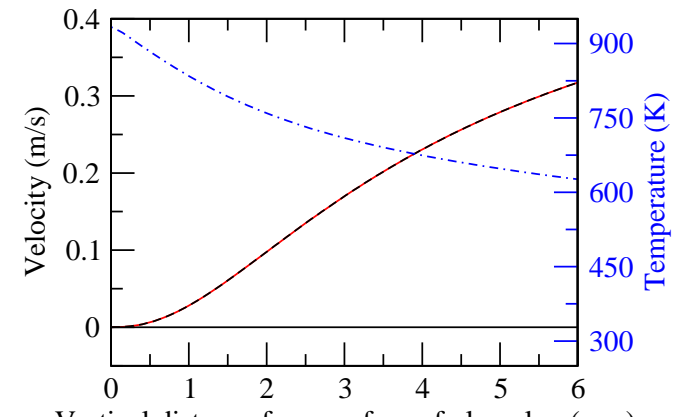

Vertical distance from surface of glowplug $(\mathrm{mm})$

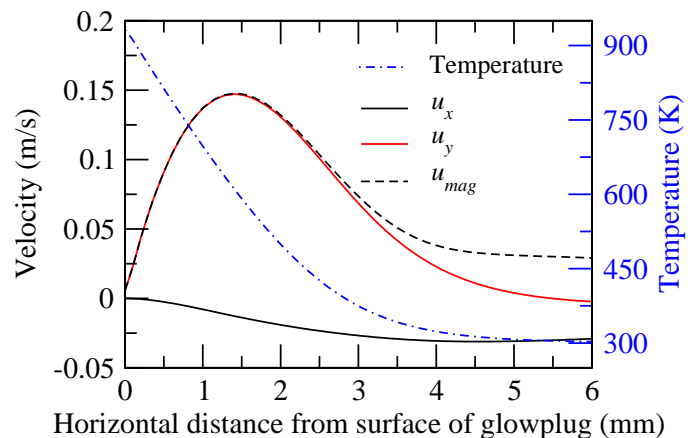

Horizontal distance from surface of glowplug $(\mathrm{mm})$

Figure 6: Top: temperature and velocity magnitude field in the vicinity of the glowplug, temperature isocontours and velocity vectors. Bottom: spatial distribution of velocity and temperature at top -from $\left(x_{o}=0 \mathrm{~mm}, y_{o}=9.3 \mathrm{~mm}\right)$ to $\left(x_{f}=0 \mathrm{~mm}, y_{f}=15.3 \mathrm{~mm}\right)$, and side -from $\left(x_{o}=2.55 \mathrm{~mm}, y_{o}=5 \mathrm{~mm}\right)$ to $\left(x_{f}=8.55 \mathrm{~mm}, y_{f}=5 \mathrm{~mm}\right)$.

the glow plug where the gas is practically at rest. The gas continues to rise to the top of the combustion vessel, turns and creates a rather complex vortical flow field (not visible on the velocity field). Further details of this flow field, and a thorough study of the ignition dynamics for $n$-hexane-air mixtures using simplified chemistry was performed by Melguizo-Gavilanes et al. [19].

In Fig. 6 (Bottom), the horizontal $\left(u_{x}\right)$ and vertical $\left(u_{y}\right)$ components of the velocity vector, black and red solid lines respectively, magnitude ( $u_{m a g}$ - dashed line), and temperature (blue dashed-dotted line) confirm that at up to $0.5 \mathrm{~mm}$ away from the 
top surface of the glowplug the flow is essentially stagnant. As a result, the gas can be readily heated by the hot surface because convective losses are minimal in this region. This plot also shows the temperature distribution of the thermal plume up to $6 \mathrm{~mm}$ away from the glowplug surface. At the side of the glowplug $(x=2.55 \mathrm{~mm}$, $y=5 \mathrm{~mm}$ ), right plot on Fig. 6 (Bottom), the temperature and velocity magnitude plots show the thermal and hydrodynamic boundary layer thickness, $5.5 \mathrm{~mm}$ and $4 \mathrm{~mm}$, respectively. The negative values of $u_{x}$ (gas moving left) display how parcels of fluid are brought into the thermal boundary layer from colder regions away from the glowplug, slowed as they approach the hot surface, changing direction gradually (see increase in $u_{y}$ ), subsequently reaching a maximum, immediately followed by a decrease to zero velocity at the wall consistent with the no-slip condition.

\subsection{Time to ignition}

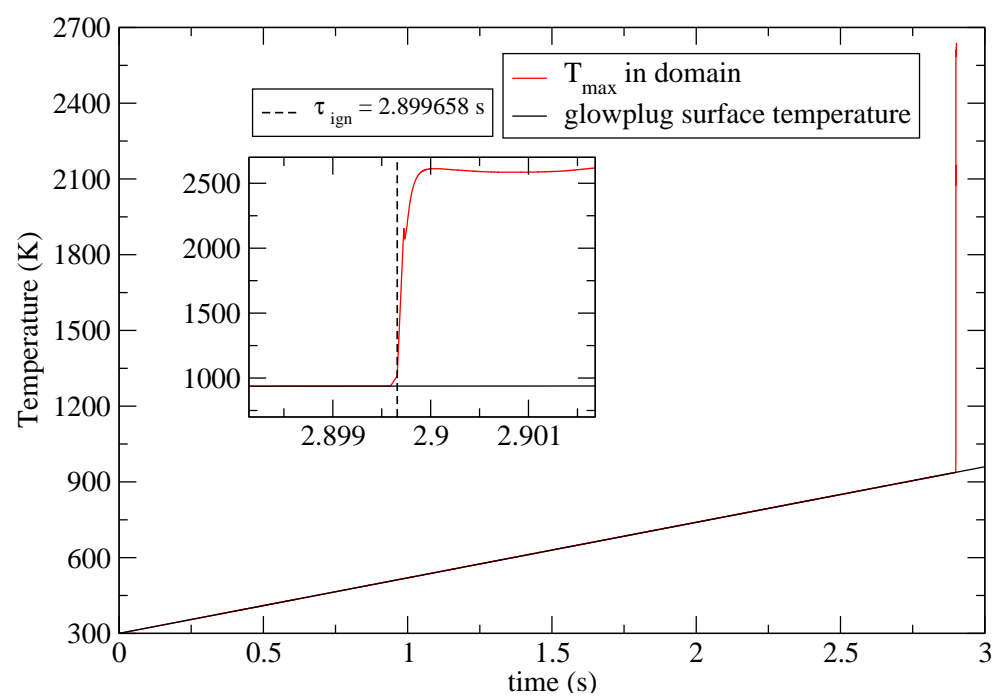

Figure 7: Temperature maximum in computational domain during the course of the simulation. Inset: closeup to ignition event.

To accurately determine the ignition time, $\tau_{i g n}$, the temperature maximum in the computational domain and glowplug surface temperature are monitored during the simulation. For the present study, ignition is defined as the time at which the maximum temperature in the domain reaches $150 \mathrm{~K}$ above the glowplug surface temperature. The rapid change in temperature during the ignition transient makes 
the ignition time insensitive to the choice of threshold temperature. The inset in Fig. 7 shows a close up of the main heat release event. The time to ignition is $\tau_{\text {ign }}=2.899658 \mathrm{~s}$, and the temperature of the glowplug surface is $\sim 938 \mathrm{~K}$ at this time. The strong dependence of ignition threshold on temperature is consistent with results from constant volume explosion computations for stoichiometric hydrogenair mixtures. These computations show a rapid increase in ignition delay times for temperatures below $1000 \mathrm{~K}$, indicative of a sharp change in activation energy in this temperature range. For example, the constant volume ignition delay time at $900 \mathrm{~K}$ is $94 \mathrm{~ms}$ decreasing to $2.09 \mathrm{~ms}$ at $930 \mathrm{~K}$. Finally, the ignition threshold predicted numerically is lower than that determined experimentally. The difference between predicted and observed ignition temperature is $10.83 \%$ and $8.75 \%$ for slow and fast heating rates, respectively. Taking into account uncertainties (upper and lower bounds) the difference is of $15 \%$ and $6.2 \%$ for $18 \mathrm{~K} / \mathrm{s}$, and $13.1 \%$ and $4 \%$ for $190 \mathrm{~K} / \mathrm{s}$. It has been reported in previous hot surface ignition work [22, 23] using stationary laser heated spheres, that surface reactions could be responsible for higher ignition thresholds than those obtained with inert materials. The effect of material/surface chemistry on ignition thresholds, while important for safety applications, is beyond the scope of the present study and will be a topic of future work.

\subsubsection{Ignition process}

Figure 8 shows velocity, temperature and $\mathrm{OH}$ mass fraction fields together with velocity vectors at four instances during the simulation. At $t=\tau_{\text {ign }}-83 \mu$ s chemical activity is already taking place at the top of the glowplug where the temperature is highest, and convective losses are minimal. The temperature maximum in the domain $(T=938 \mathrm{~K})$ corresponds to that of the glowplug surface until ignition takes place. At $t=\tau_{i g n}+167 \mu \mathrm{s}$, an ignition center appears on the temperature field as closed contours at the top surface of the glowplug. The temperature contours are rescaled to cover the full range of temperature within the computational domain at each time shown in Fig. 8. This ignition center is accompanied by a localized increase in $\mathrm{OH}$ concentration (by almost 5 orders of magnitude), and gas velocity. 


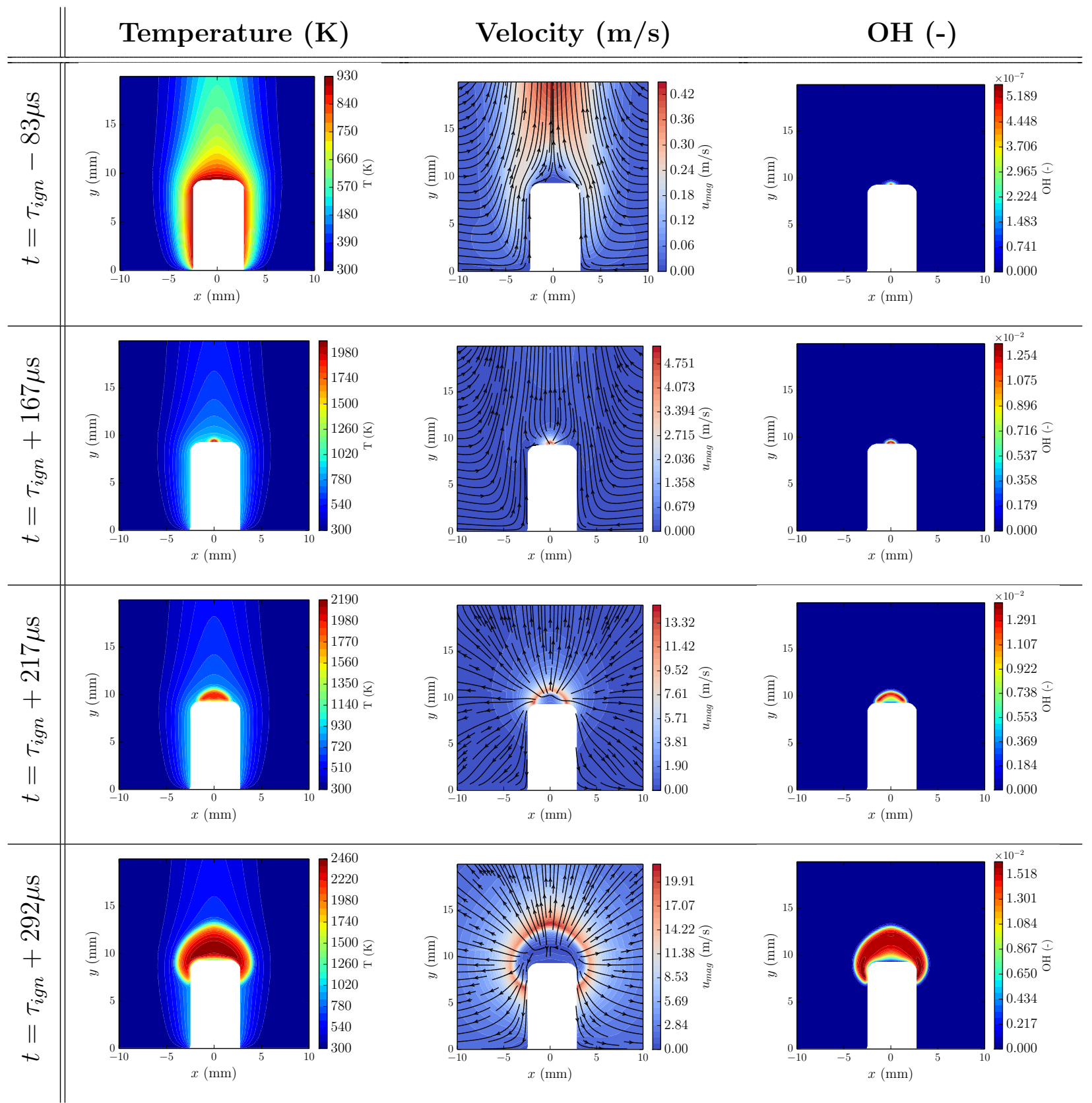

Figure 8: Temperature (left), velocity magnitude (middle) and $\mathrm{OH}$ mass fraction (right) fields during the ignition sequence. All fields are rescaled at every time shown.

The velocity vectors show how the ignition kernel abruptly expands and pushes away the surrounding gas. Further acceleration of the gas from $4.82 \mathrm{~m} / \mathrm{s}$ to $13.8 \mathrm{~m} / \mathrm{s}$ in $50 \mu \mathrm{s}$ can be seen on the velocity fields at $t=\tau_{i g n}+217 \mu \mathrm{s}$. The rapid expansion is evidenced by the abrupt change in direction of the velocity vectors. A nascent flame is observed in the temperature contours, and the fuel is nearly completely consumed within the flame kernel. The mass fraction of $\mathrm{H}_{2} \mathrm{O}$ is 0.235 , close to the 
theoretical value of 0.255 given by complete oxidation of a stoichiometric mixture (not shown in Fig. 8). The last frame, $t=\tau_{i g n}+292 \mu \mathrm{s}$, shows the early stages of flame propagation. The surrounding gas is displaced radially outwards due to the volumetric expansion of the burned products. The shape of the flame is determined by the preferential propagation of the combustion front along the thermal plume where the fresh combustible mixture is hottest. The temperature contours show clearly the high-temperature region in the combustion products. The simulations reproduce the same behavior observed in the experiments reported in section 4.1 with the ignition kernel forming at the top of the glowplug followed by flame propagation (see Fig. 5).

\section{Discussion}

\subsection{Energy equation analysis}

To gain additional insight into the processes taking place at the top of the glowplug, each of the terms in the energy conservation equation is plotted along the vertical centerline from the surface of the glowplug (see Fig. 9). The plots are taken at the same times as in Figure 8 to allow for a direct comparison. The abscissas represent the vertical distance along the axis of symmetry measured from the top surface of the glowplug, whereas the ordinates show the corresponding energy density and temperature. The solid lines are the convective and diffusive heat losses, and the chemical source term given respectively by $h_{\text {Convection }}=-\nabla \cdot\left(\rho \mathbf{u} h_{s}\right)$, $h_{\text {Diffusion }}=\nabla \cdot\left(\kappa / c_{p} \nabla h_{s}\right)$, and $h_{\text {Source }}=\dot{q}_{\text {chem }}$. The dashed line is the sum of the above terms, $h_{\text {Unsteady }}$, and the dashed-dotted line is the temperature. Shortly before ignition (Fig. 9 top left), close to the glowplug surface, the source term is mostly balanced by diffusion. The sum is positive up to $0.5 \mathrm{~mm}$ from the glowplug surface, and the temperature maximum remains at the wall. Further away from the glowplug's wall $(0.5-5 \mathrm{~mm})$, convection balances diffusion.

In Fig. 9 top right, $250 \mu$ s later, the temperature maximum is no longer at the wall but roughly $0.12 \mathrm{~mm}$ away from the surface of the glowplug, hence, the rate at which heat is diffused back to the wall is not large enough to counteract the 

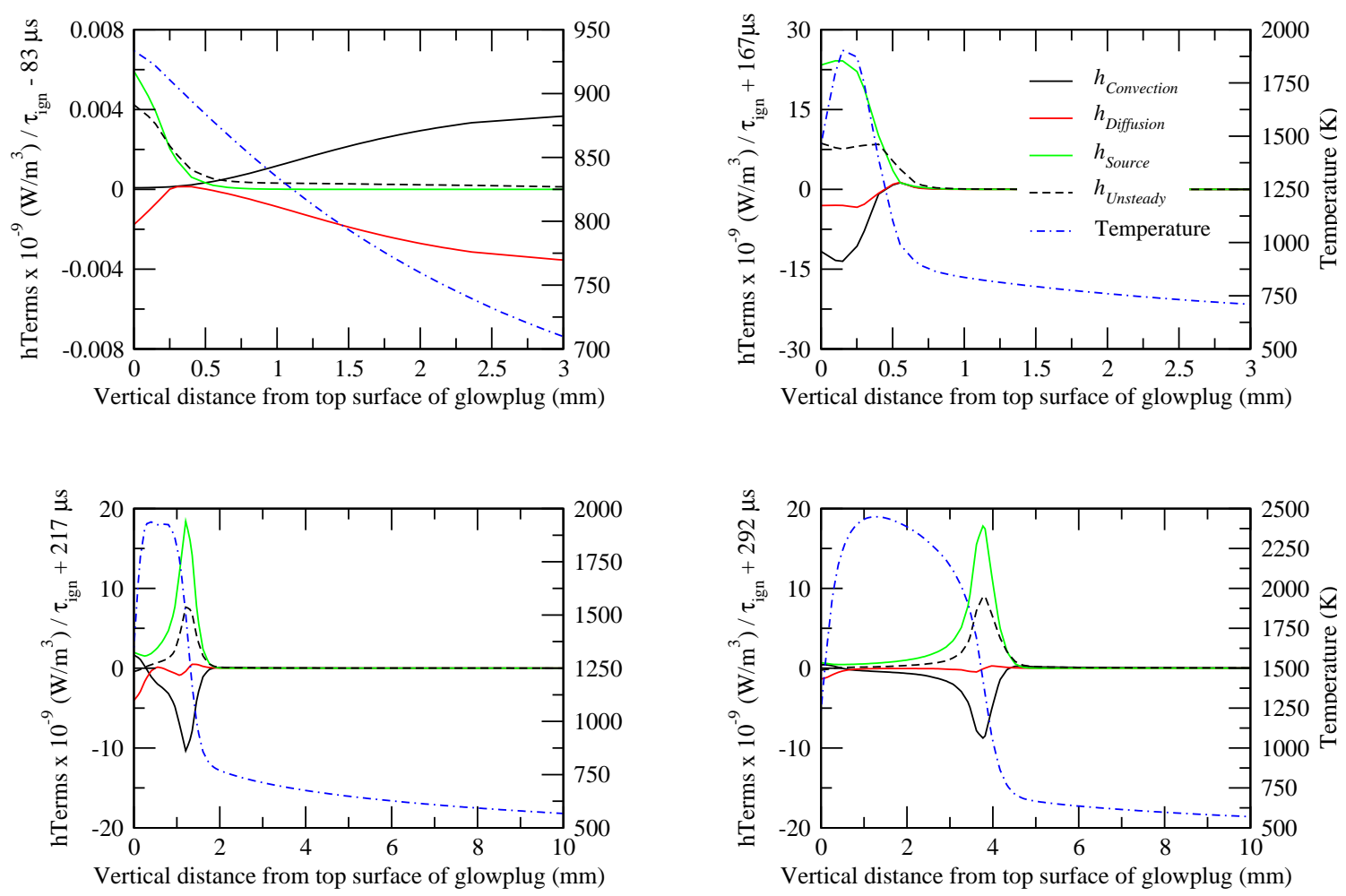

Figure 9: Ignition process: contributions of each term in energy equation and temperature along normal distance from top surface of the glowplug. Top Left: at $t=\tau_{i g n}-83 \mu \mathrm{s}$ - shortly before ignition. Top Right: at $t=\tau_{\text {ign }}+167 \mu \mathrm{s}$ - ignition kernel formation. Bottom Left: at $t=\tau_{\text {ign }}+217 \mu \mathrm{s}$ - flame kernel formation. Bottom right: at $t=\tau_{i g n}+292 \mu \mathrm{s}$ - early stages of flame propagation.

rate at which heat is released by the chemistry at this location, signaling the birth of an ignition center. The explosive nature of the ignition event can be visualized in the increase in the source term from $4 \times 10^{6} \mathrm{~W} / \mathrm{m}^{3}$ to $22.5 \times 10^{9} \mathrm{~W} / \mathrm{m}^{3}$ over $250 \mu \mathrm{s}$. The structure of an incipient ignition center can also be observed. In Fig. 9 bottom left, an expanding flame kernel is captured. Due to the abrupt expansion of the gas, and associated velocity $(13.8 \mathrm{~m} / \mathrm{s})$, the chemical source term is balanced at the flame front mostly by the convective term. At the wall, the balance is maintained by diffusion as expected. The plot at the bottom right of Fig. 9, displays very clearly the structure of the flame propagating away from the surface of the glowplug. 


\subsection{Chemical activity above the glowplug, and chemical pathways}

Plotting the contributions of each term in the energy equation and temperature perpendicularly from the top surface of the glowplug was a necessary step to find the location where ignition takes place, which is centered around the point $(x=0 \mathrm{~mm}$, $y=9.42 \mathrm{~mm}$ ), about $0.12 \mathrm{~mm}$ above the glowplug. To investigate the chemistry in more detail, temporal data at the ignition location were collected for temperature, chemical source term, diffusive and convective losses, together with species profiles. The plots in Fig. 10, show the start of heat release as early as $t=2.8996 \mathrm{~s}$, at that time diffusion and convection immediately counteract the source term. The ignition event is marked simultaneously by the sudden increase in the rate at which energy is deposited in the gas, a rapid rise in gas temperature, a rapid consumption of fuel and the production of $\mathrm{H}_{2} \mathrm{O}$ and radical species, $\mathrm{H}, \mathrm{OH}$ and $\mathrm{O}$. Before ignition takes place, at $t=2.8985 \mathrm{~s}$ the species $\mathrm{H}_{2} \mathrm{O}_{2}$ and $\mathrm{HO}_{2}$ have already started to build up. At $t=2.8995 \mathrm{~s}, \mathrm{HO}_{2}$ increases substantially just before ignition occurs.

Figure 11 displays the reaction pathway diagram at a location just above the glowplug. The chain branching reactions, $\mathrm{R}_{1}: \mathrm{H}+\mathrm{O}_{2}=\mathrm{OH}+\mathrm{O}$ and $\mathrm{R}_{2}: \mathrm{O}+\mathrm{H}_{2}$ $=\mathrm{OH}+\mathrm{H}$, account only for 15 to $20 \%$ of the reactants consumption. Molecular hydrogen and oxygen are mostly consumed by $\mathrm{R}_{3}: \mathrm{H}_{2}+\mathrm{OH}=\mathrm{H}_{2} \mathrm{O}+\mathrm{H}$ and $\mathrm{R}_{4}$ : $\mathrm{H}+\mathrm{O}_{2}(+\mathrm{M})=\mathrm{HO}_{2}(+\mathrm{M})$, respectively. While $\mathrm{R}_{3}$ is highly exothermic and induces significant release of energy, $\mathrm{R}_{4}$ delays the formation of $\mathrm{OH}$ radical. Only $34 \%$ of $\mathrm{OH}$ is formed by $\mathrm{R}_{1}$ and $\mathrm{R}_{2}$, whereas the sequence $\mathrm{R}_{4}$ followed by $\mathrm{R}_{5}: \mathrm{HO}_{2}+\mathrm{H}=$ $\mathrm{OH}+\mathrm{OH}$ represents $66 \%$ of $\mathrm{OH}$ formation. Under this low temperature conditions, indirect formation of $\mathrm{OH}$ radicals through linear chain chemical processes dominates over direct, chain branching reactions.

\section{Conclusion}

Two-dimensional simulations of ignition by a transiently heated commercial glowplug were performed. In agreement with experiments, ignition was observed to occur at the top of the glowplug. The details of the ignition kernel evolution was explained 

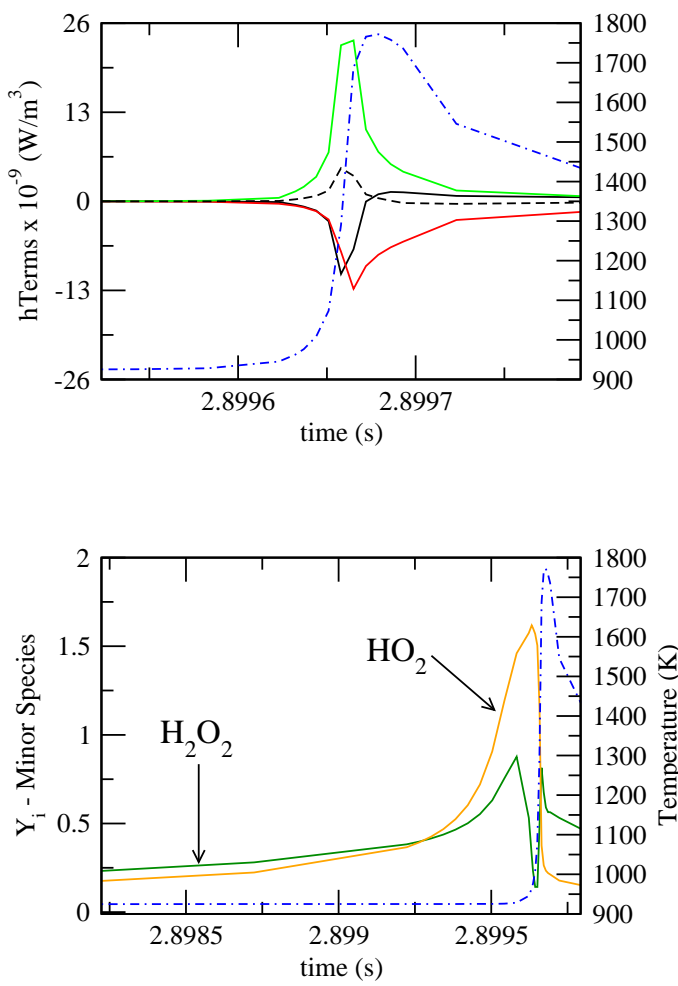
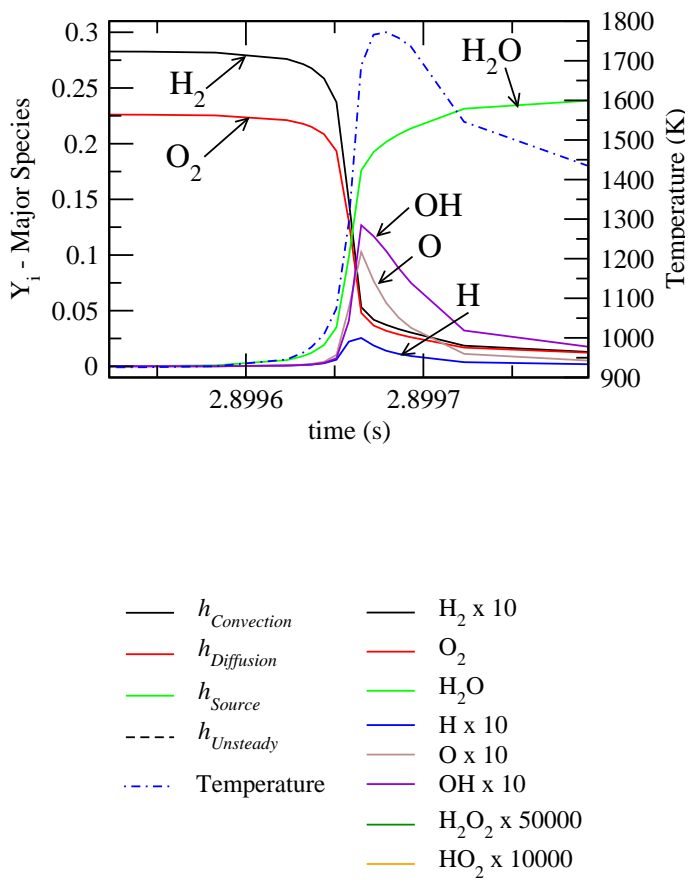

Figure 10: Temporal evolution of each term in energy equation, temperature and species mass fractions at the ignition location, $(x=0 \mathrm{~mm}, y=9.42 \mathrm{~mm}), 0.12 \mathrm{~mm}$ above the glowplug.

using selected velocity, species and temperature fields from the simulations. Additional insight was achieved by analyzing the individual contributions of the terms in the energy conservation equation. Close to the wall, diffusion counteracts the heat release due to the chemistry, whereas far away, convection and diffusion maintain the balance. Significant chemical activity starts when the mixture temperature in the separated region above the glowplug rises over $930 \mathrm{~K}$; the parcels of gas in the stagnation volume have a long enough residence time for reactions to cause exponential growth of radical and intermediate species in this temperature range. At $0.12 \mathrm{~mm}$ normal to the top of the glowplug surface, the heat release rate is greater than the rate at which heat is diffused back to the wall giving birth to an ignition kernel.

The overall quantitative agreement between the numerical predictions and experiments in terms of surface temperature at ignition, ignition location and flow field features in the vicinity of the hot surface demonstrate the ability of our numerical 


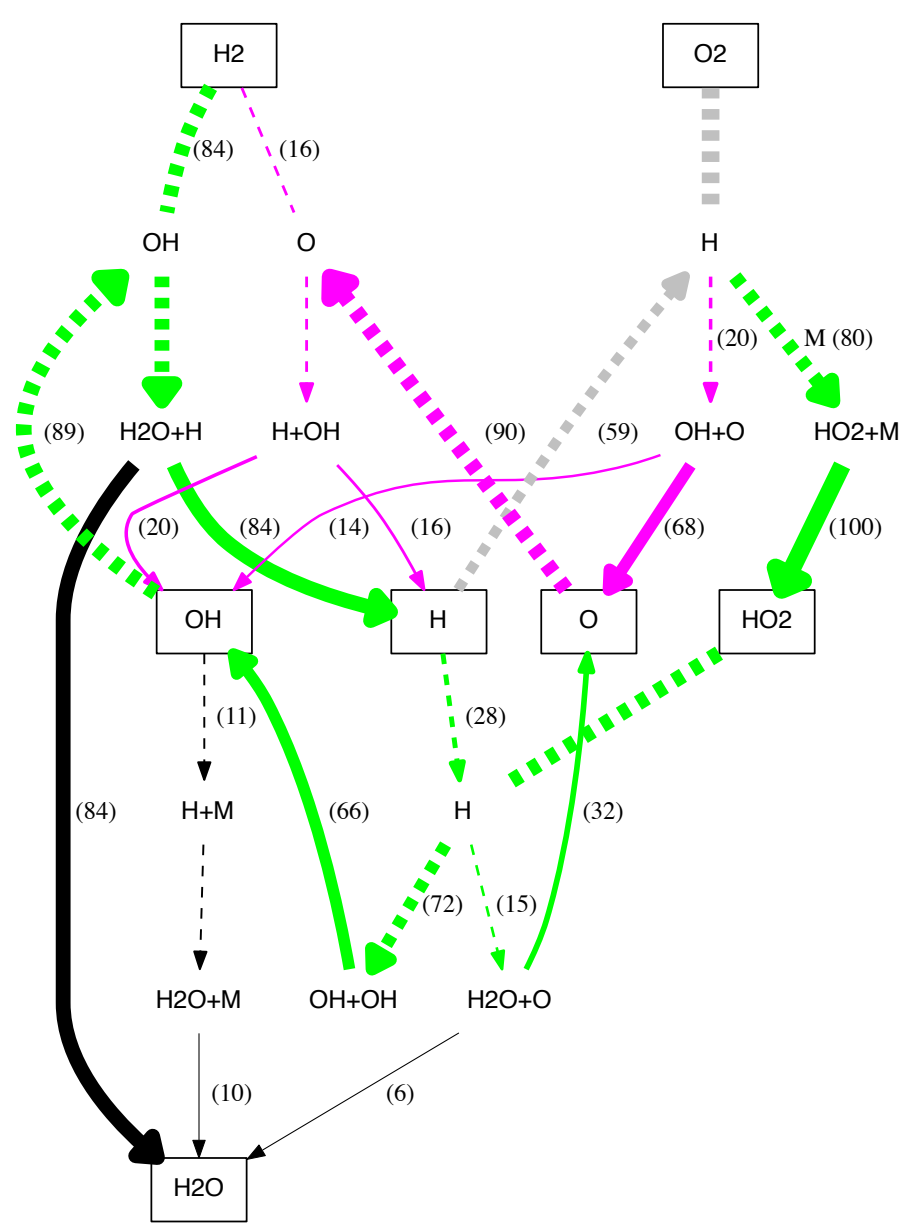

Figure 11: Reaction pathway analysis at the ignition location, $(x=0 \mathrm{~mm}, y=9.42 \mathrm{~mm}), 0.12 \mathrm{~mm}$ above the top surface of the glowplug. Boxes represent species reservoirs, solid lines are reservoir inputs, and dashed lines are reservoirs outputs. Green: non chain-branching pathways; Magenta: chain-branching pathways; Gray: mixed pathways.

model to reproduce important aspects of the ignition process. Furthermore, these results show the importance of flow separation in creating zones that are prone to ignition: convective transport of energy and species out of the separated region is minimized, and the build up of intermediate species $\left(\mathrm{HO}_{2}\right.$ and $\left.\mathrm{H}_{2} \mathrm{O}_{2}\right)$ can only be opposed by diffusion, facilitating the fast production of $\mathrm{OH}$ and subsequent coupled branching chain-thermal run away characteristic of ignition events.

Under these thermodynamic conditions, the reaction pathway analysis showed that ignition is essentially driven by a linear chain chemical process. The chain branching reactions, $\mathrm{H}+\mathrm{O}_{2}=\mathrm{OH}+\mathrm{O}$ and $\mathrm{O}+\mathrm{H}_{2}=\mathrm{OH}+\mathrm{H}$ constitute minor pathways in producing reactive radicals. The main sequence leading to hydroxyl 
radical formation is: $\mathrm{H}+\mathrm{O}_{2}(+\mathrm{M})=\mathrm{HO}_{2}+\mathrm{M} ; \mathrm{HO}_{2}+\mathrm{H}=\mathrm{OH}+\mathrm{OH}$.

We conclude that quantitative predictions of ignition thresholds for hot surfaces are possible using a detailed simulation that includes correct initial and boundary conditions to capture important features such as boundary layer separation, and energy transport processes. Including additional physics such as surface chemistry (i.e. adsorption of reactive species and/or catalytic effects), as well as a systematic variation of experimental parameters (i.e. hot surface size, material, mixture equivalence ratio, etc.) and their effect on ignition thresholds are potentially important but were outside of the scope of this study and remain to be investigated in detail.

\section{Acknowledgments}

This work was carried out in the Explosion Dynamics Laboratory of the California Institute of Technology. J. Melguizo-Gavilanes was supported by the Natural Sciences and Engineering Research Council of Canada (NSERC) Postdoctoral Fellowship Program. L. Boeck, R. Mével and J.E. Shepherd by The Boeing Company through a Strategic Research and Development Relationship Agreement CT-BAGTA-1. This work used the Extreme Science and Engineering Discovery Environment (XSEDE), which is supported by National Science Foundation grant number ACI-1053575.

\section{References}

[1] Coward, H.F. and Guest, P.G., Ignition of natural gas-air mixtures by heated metal bars 1, Journal of the American Chemistry Society, 1927, 49(10), pp. 2479-2486

[2] Kuchta, J.M., Investigation of fire and explosion accidents in the chemical, mining, and fuel related industries, 1985, Bulletin 680, Bureau of Mines.

[3] Brabrauskas, V., Ignition Handbook, 2003, Fire Science Publishers.

[4] Boettcher, P.A., Thermal Ignition, 2012, Ph.D Thesis. Caltech. 
[5] Gray, B.F., The dependence of spontaneous ignition temperature on surface to volume ratio in static systems for fuels showing negative temperature coefficient, Combustion and Flame, 1970, 14(1), pp. 113-115

[6] Laurendeau, N.M., Thermal ignition of methane-air mixtures by hot surfaces: A critical examination, Combustion and Flame, 1982, 46, pp. 29-49

[7] Kumar, R.K., (1989). Ignition of hydrogen-oxygen-diluent mixtures adjacent to a hot, nonreactive surface, Combustion and Flame, 1989, 75(2), pp. 197-215

[8] Adler, J., Ignition of a combustible stagnant gas layer by a circular hot spot. Combustion Theory and Modeling, 1999, 3(2), pp. 359-369

[9] Boettcher, P.A., Mével, R., Thomas, V. and Shepherd, J.E., (2012). The effect of heating rates on low temperature hexane combustion, Fuel, 2012, 96, pp. $392-403$

[10] Poinsot, T. and Veynante, D., Theoretical and Numerical Combustion, 2005, Edwards.

[11] Smooke, M. D., The computation of laminar flames, Proceedings of the Combustion Institute, 2013, 34, pp. 65-98

[12] Weller, H.G., Tabor, G., Jasak, H., and Fureby, C., A tensorial approach to continuum mechanics using object-oriented techniques, Journal of Computational Physics, 1998, 12, pp. 620-631

[13] Saad, Y., Iterative methods for sparse linear systems, 2003, Society for Industrial and Applied Mathematics, Philadelphia.

[14] Demirdzic, I., Lilek, Z. and Péric, M. (1993). A collocated finite volume method for predicting flows at all speeds. Int. J. Numer. Meth. Fl., 16, 1029-1050.

[15] Oran, E.S. and Boris, J.P., Numerical Simulation of Reactive Flow, 2001, Cambridge University Press. 
[16] Mével, R., Javoy, S., Lafosse, F., Chaumeix, N., Dupré, G. and Paillard, C.E., Hydrogen-nitrous oxide delay time: shock tube experimental study and kinetic modelling, Proceedings of The Combustion Institute, 2009, 32, pp. 359-366.

[17] Mével, R., Javoy, S. and Dupré, G., A chemical kinetic study of the oxidation of silane by nitrous oxide, nitric oxide and oxygen, Proceedings of The Combustion Institute, 2011, 33, pp. 485-492.

[18] Goodwin, D. Cantera: object-oriented software for reacting flows. Technical Report, California Institute of Technology, 2005.

[19] Melguizo-Gavilanes, J., Nové-Josserand, A., Coronel, S., Mével R. and Shepherd, J.E. (2015) Hot surface ignition of $n$-hexane mixtures using simplified kinetics. Combust. Sci. Techology, Accepted

[20] Nestor, O. H. and Olsen, H. N. (1960). Numerical Methods for Reducing Line and Surface Probe Data. SIAM Rev., 3(2):200-207.

[21] Menon, S., Boettcher, P., Ventura, B., Blanquart, G. (2016) Hot surface ignition of n-hexane in air, Combustion and Flame, 163, 42-53.

[22] Beyer, M. and Markus, D., Ignition of explosive atmospheres by small hot particles : Comparison of experiments and simulations, Science and Technology of Energetic Materials, $\mathbf{2 0 1 2}$

[23] Roth, D., Sharma, P., Haeber, T., Schiessl, R., Bockhorn, H., Maas, U., Beyer, M. and Markus, D., Ignition by Mechanical Sparks: Ignition of Hydrogen/Air Mixtures by Submillimeter-Sized Hot Particles, Combustion Science and Technology, 2014, 186(10-11), pp. 1606-1617 